\title{
Accurate and versatile modeling of electromagnetic scattering on periodic nanostructures with a surface integral approach
}

\author{
Benjamin Gallinet, ${ }^{1, *}$ Andreas M. Kern, ${ }^{1}$ and Olivier J. F. Martin ${ }^{1,2}$ \\ ${ }^{1}$ Nanophotonics and Metrology Laboratory, Swiss Federal Institute of Technology Lausanne (EPFL), \\ 1015 Lausanne, Switzerland \\ ${ }^{2}$ E-mail: olivier.martin@epfl.ch \\ *Corresponding author: benjamin.gallinet@epfl.ch
}

Received May 28, 2010; revised July 21, 2010; accepted August 5, 2010;

posted August 6, 2010 (Doc. ID 129200); published September 27, 2010

\begin{abstract}
A surface integral formulation for light scattering on periodic structures is presented. Electric and magnetic field equations are derived on the scatterers' surfaces in the unit cell with periodic boundary conditions. The solution is calculated with the method of moments and relies on the evaluation of the periodic Green's function performed with Ewald's method. The accuracy of this approach is assessed in detail. With this versatile boundary element formulation, a very large variety of geometries can be simulated, including doubly periodic structures on substrates and in multilayered media. The surface discretization shows a high flexibility, allowing the investigation of irregular shapes including fabrication accuracy. Deep insights into the extreme near-field of the scatterers as well as in the corresponding far-field are revealed. This method will find numerous applications for the design of realistic photonic nanostructures, in which light propagation is tailored to produce novel optical effects. (C) 2010 Optical Society of America
\end{abstract}

OCIS codes: $050.1755,350.4238$.

\section{INTRODUCTION}

The study of periodic structures has been the subject of great interest in diffraction gratings [1]. It finds nowadays numerous other applications in nanophotonics, especially photonic bandgap materials [2], metamaterials [3], or plasmonic crystals [4]. This interest is accompanied by an increasing demand for efficient modeling tools that can handle actual experimental and fabrication conditions. Among the most popular numerical methods, the Fouriermodal [5] and plane-wave expansion [6] methods, or the rigorous coupled-wave analysis [7], although rather straightforward to implement remain efficient only for specific types of geometry. Scattering matrix $[8,9]$ methods are well suited for calculating reflectivity or emission spectra in photonic structures. Methods based on local formulations, such as the finite-difference time-domain method [10] or the finite-element method (FEM) [11,12], are more flexible but do not satisfy Sommerfeld radiation conditions. Therefore boundary conditions must be imposed at the edges of the computation window. The FEM is proven very accurate, can handle realistic structures, and produces sparse matrices efficiently solved with appropriate algorithms [12]. Although the focus of the present work is on general three-dimensional (3D) periodic systems, one should mention the vast body of work developed for diffraction gratings since the early theory of Rayleigh: the integral and differential theories [1], the Chandezon method [13], the modal formulation [14], or methods based on finite elements [15].

Integral equation methods are multiscale and well suited for electromagnetic scattering problems since only the discretization of the scatterer is necessary and boundary conditions are rigorously included in the calculations. Volume integral equation (VIE) methods include the discrete dipole approximation (DDA) [16] and the Green's tensor technique [17], both widespread in the nanophotonics community. The DDA method has been generalized to a periodic array of scatterers $[18,19]$ and more recently to periodic arrays embedded in a multilayer system [20]. However, VIE methods result in full matrices with high cost in memory and computational time, including the evaluation of the periodic Green's function. For nonhomogeneous scatterers hybrid finite-element boundaryintegral methods are proven very efficient and popular for microwaves [12,21].

With surface integral equation (SIE) methods [22-25] only the scatterer's surface is discretized. Although SIE methods generate dense matrices, the fact that they scale with only the second power of the lateral dimension makes them very efficient for homogeneous scatterers. Very popular in the microwave community, SIE methods based on the method of moments (MoM) [12,26] have also been extensively used in the microwave regime for periodic lossy [21,27-29] or metallic [30] systems. Recently, it has been successfully introduced to optics to simulate individual high permittivity and plasmonic scatterers [25]. Let us point out that this semi-analytical formulation can give insights into the extreme near-field behavior, even for rapidly changing fields, as well as into the corresponding far-field radiation. This is important since state-ofthe-art optical measurement techniques offer the possibility to study optical far-field properties as well as to 
provide a deep insight into the corresponding near-field physics [31]. Furthermore, the MoM discretization shows a high flexibility, allowing, i.e., the investigation of fabrication accuracy.

It is the purpose of the present paper to develop a MoM-based SIE formulation applicable to arbitrary periodic nanostructures. The evaluation of the periodic Green's function is performed with Ewald's method, and an implementation of specific periodic boundary conditions at the edges of the unit cell is described. The formulation can be applied to an arbitrary number of regions and composite objects. A broad variety of geometries close to experimental and fabrication conditions can be simulated, in particular doubly periodic structures on substrates and in multilayered media.

The paper is organized as follows: in Section 2 the SIEs for periodic systems are derived. Their numerical implementation using the MoM and Ewald's method is described in Section 3. The method is illustrated and its accuracy is assessed with three examples in Section 4. For infinitely extending high contrast dielectric and dielectric-metal planar interfaces, numerical results are compared to an analytical solution. The photonic bandgap properties of a square array of pillars and how they are affected by a substrate are investigated. As a final example, some transmission and reflection calculations in a fishnet metamaterial reveal a negative refractive index in the red part of the optical spectrum.

\section{SURFACE INTEGRAL EQUATIONS FOR PERIODIC SYSTEMS}

In this section, the SIEs for electromagnetic scattering are first derived for infinite 3D space. Periodic systems are then discussed, resulting into integral equations for the unit cell with periodic boundary conditions.

The 3D space describing our scattering system is considered to be divided into $N$ different regions $V_{n}, n$ $=1, \ldots, N$ with dielectric permittivity $\epsilon_{n}$ and magnetic permeability $\mu_{n}$ (Fig. 1). A harmonic time-dependence for

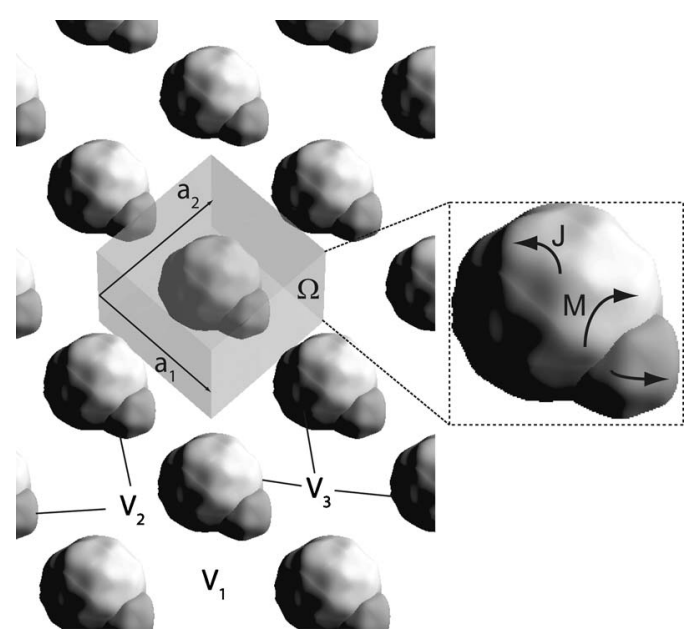

Fig. 1. Space division into regions $V_{n}$ with dielectric permittivity $\epsilon_{n}$ and magnetic permeability $\mu_{n}$. The regions have the symmetry of a lattice with unit cell $\Omega$ and primitive lattice vectors $\mathbf{a}_{i}$. Equivalent surface currents $\mathbf{J}$ and $\mathbf{M}$ flow on the scatterer's surfaces. the fields $\mathbf{U}(\mathbf{r}, t)=\mathbf{U}_{0}(\mathbf{r}) e^{-i \omega t}$ is assumed throughout this paper. The electric field $\mathbf{E}$ in each region must satisfy the equation

$$
\nabla \times \nabla \times \mathbf{E}(\mathbf{r})-k_{n}^{2} \mathbf{E}(\mathbf{r})=i \omega \mu_{n} \mathbf{j}(\mathbf{r}), \quad \mathbf{r} \in V_{n},
$$

where $k_{n}^{2}=\omega^{2} \epsilon_{n} \mu_{n}$ is the wavenumber for electromagnetic waves in region $V_{n}$ and $\mathbf{j}$ denotes the volume current density. A dyadic Green's function $\underline{\underline{G}}_{n}$ for region $V_{n}$ is introduced:

$$
\nabla \times \nabla \times \underline{\underline{G}}_{n}\left(\mathbf{r}, \mathbf{r}^{\prime}\right)-k_{n}^{2} \underline{\underline{G}}_{n}\left(\mathbf{r}, \mathbf{r}^{\prime}\right)=\underline{\underline{1}} \delta\left(\mathbf{r}-\mathbf{r}^{\prime}\right) .
$$

Multiplying Eq. (1) by $\underline{\underline{G}}_{n}\left(\mathbf{r}, \mathbf{r}^{\prime}\right)$ from the right and Eq. (2) by $\mathbf{E}(\mathbf{r})$ from the left and subtracting the two equations, one obtains

$$
\begin{aligned}
& {[\nabla \times \nabla \times \mathbf{E}(\mathbf{r})] \cdot \underline{\underline{G}}_{n}\left(\mathbf{r}, \mathbf{r}^{\prime}\right)-\mathbf{E}(\mathbf{r}) \cdot\left[\nabla \times \nabla \times \underline{\underline{G}}_{n}\left(\mathbf{r}, \mathbf{r}^{\prime}\right)\right]} \\
& \quad=i \omega \mu_{n} \mathbf{j}(\mathbf{r}) \cdot \underline{\underline{G}}_{n}\left(\mathbf{r}, \mathbf{r}^{\prime}\right)-\mathbf{E}(\mathbf{r}) \delta\left(\mathbf{r}-\mathbf{r}^{\prime}\right) .
\end{aligned}
$$

Integrating Eq. (3) over $V_{n}$ and transforming its left-hand side following Eq. (A.45) in [32] leads to

$$
\begin{gathered}
\int_{V_{n}} \mathrm{~d} V \nabla \cdot\left([\nabla \times \mathbf{E}(\mathbf{r})] \times \underline{\underline{G}}_{n}\left(\mathbf{r}, \mathbf{r}^{\prime}\right)+\mathbf{E}(\mathbf{r}) \times\left[\nabla \times \underline{\underline{G}}_{n}\left(\mathbf{r}, \mathbf{r}^{\prime}\right)\right]\right) \\
=i \omega \mu_{n} \int_{V_{n}} \mathrm{~d} V \mathbf{j}(\mathbf{r}) \cdot \underline{\underline{G}}_{n}\left(\mathbf{r}, \mathbf{r}^{\prime}\right)- \begin{cases}\mathbf{E}\left(\mathbf{r}^{\prime}\right): & \mathbf{r}^{\prime} \in V_{n} \\
0: & \text { otherwise. }\end{cases}
\end{gathered}
$$

The integral on the right-hand side can be identified to the incident electric field $\mathbf{E}_{n}^{\text {inc }}$ generated by the electrical current density $\mathbf{j}$ inside $V_{n}$ :

$$
\begin{aligned}
i \omega \mu_{n} \int_{V_{n}} \mathrm{~d} V \mathbf{j}(\mathbf{r}) \cdot \underline{\underline{G}}_{n}\left(\mathbf{r}, \mathbf{r}^{\prime}\right) & =i \omega \mu_{n} \int_{V_{n}} \mathrm{~d} V \underline{\underline{G}}_{n}\left(\mathbf{r}^{\prime}, \mathbf{r}\right) \cdot \mathbf{j}(\mathbf{r}) \\
& =\mathbf{E}_{n}^{\text {inc }}\left(\mathbf{r}^{\prime}\right),
\end{aligned}
$$

where we used the reciprocity of the dyadic Green's function $\underline{\underline{G}}_{n}\left(\mathbf{r}, \mathbf{r}^{\prime}\right)^{T}=\underline{\underline{G}}_{n}\left(\mathbf{r}^{\prime}, \mathbf{r}\right)$ The integral on the left-hand side of Eq. (4) can be transformed into a surface integral on the boundary $\partial V_{n}$ of region $V_{n}$ using Gauss' theorem:

$$
\begin{aligned}
\int_{\partial V_{n}} \mathrm{~d} S \hat{\mathbf{n}}_{n}(\mathbf{r}) \cdot\left([\nabla \times \mathbf{E}(\mathbf{r})] \times \underline{\underline{G}}_{n}\left(\mathbf{r}, \mathbf{r}^{\prime}\right)+\mathbf{E}(\mathbf{r})\right. \\
\left.\times\left[\nabla \times \underline{\underline{G}}_{n}\left(\mathbf{r}, \mathbf{r}^{\prime}\right)\right]\right) \\
=\mathbf{E}_{n}^{\operatorname{inc}}\left(\mathbf{r}^{\prime}\right)- \begin{cases}\mathbf{E}\left(\mathbf{r}^{\prime}\right): & \mathbf{r}^{\prime} \in V_{n} \\
0: & \text { otherwise, }\end{cases}
\end{aligned}
$$

where $\hat{\mathbf{n}}_{n}$ is the outward oriented normal vector on the boundary $\partial V_{n}$. From the time-harmonic dependence of the fields and some dyadic analysis [32],

$$
\begin{aligned}
\hat{\mathbf{n}}_{n}(\mathbf{r}) \cdot\left([\nabla \times \mathbf{E}(\mathbf{r})] \times \underline{\underline{G}}_{n}\left(\mathbf{r}, \mathbf{r}^{\prime}\right)\right) \\
=\left(\hat{\mathbf{n}}_{n}(\mathbf{r}) \times[\nabla \times \mathbf{E}(\mathbf{r})]\right) \cdot \underline{\underline{G}}_{n}\left(\mathbf{r}, \mathbf{r}^{\prime}\right) \\
=i \omega \mu_{n} \underline{\underline{G}}_{n}\left(\mathbf{r}^{\prime}, \mathbf{r}\right) \cdot\left[\hat{\mathbf{n}}_{n}(\mathbf{r}) \times \mathbf{H}(\mathbf{r})\right] .
\end{aligned}
$$

On the other hand, using the reciprocity of the dyadic Green's function $\left(\nabla \times \underline{\underline{G}}_{n}\left(\mathbf{r}, \mathbf{r}^{\prime}\right)\right)^{T}=-\nabla \times \underline{\underline{G}}_{n}\left(\mathbf{r}^{\prime}, \mathbf{r}\right)$, 


$$
\begin{aligned}
\hat{\mathbf{n}}_{n}(\mathbf{r}) \cdot\left(\mathbf{E}(\mathbf{r}) \times\left[\nabla \times \underline{\underline{G}}_{n}\left(\mathbf{r}, \mathbf{r}^{\prime}\right)\right]\right) \\
=\left[\hat{\mathbf{n}}_{n}(\mathbf{r}) \times \mathbf{E}(\mathbf{r})\right] \cdot\left[\nabla \times \underline{\underline{G}}_{n}\left(\mathbf{r}, \mathbf{r}^{\prime}\right)\right] \\
=-\left[\nabla \times \underline{\underline{G}}_{n}\left(\mathbf{r}^{\prime}, \mathbf{r}\right)\right] \cdot\left[\hat{\mathbf{n}}_{n}(\mathbf{r}) \times \mathbf{E}(\mathbf{r})\right] .
\end{aligned}
$$

Introducing the equivalent surface current densities $\mathbf{J}_{n}$ $=\hat{\mathbf{n}}_{n} \times \mathbf{H}$ and $\mathbf{M}_{n}=-\hat{\mathbf{n}}_{n} \times \mathbf{E}$ defined on $\partial V_{n}$ (Fig. 1), Eq. (6) becomes

$$
\begin{aligned}
& i \omega \mu_{n} \int_{\partial V_{n}} \mathrm{~d} S^{\prime} \underline{\underline{G}}_{n}\left(\mathbf{r}, \mathbf{r}^{\prime}\right) \cdot \mathbf{J}_{n}\left(\mathbf{r}^{\prime}\right) \\
& \quad+\int_{\partial V_{n}} \mathrm{~d} S^{\prime}\left[\nabla^{\prime} \times \underline{\underline{G}}_{n}\left(\mathbf{r}, \mathbf{r}^{\prime}\right)\right] \cdot \mathbf{M}_{n}\left(\mathbf{r}^{\prime}\right) \\
& =\mathbf{E}_{n}^{\text {inc }}(\mathbf{r})- \begin{cases}\mathbf{E}(\mathbf{r}): & \mathbf{r} \in V_{n} \\
0: & \text { otherwise. }\end{cases}
\end{aligned}
$$

Taking the second case on the right-hand side of Eq. (9), the continuity of the tangential component of the fields $\mathbf{E}$ and $\mathbf{H}$ allows one to take the limit $\mathbf{r} \rightarrow \partial V_{n}$, leading to the electric field integral equation (EFIE)

$$
\begin{aligned}
& \left(i \omega \mu_{n} \int_{\partial V_{n}} \mathrm{~d} S^{\prime} \underline{\underline{G}}_{n}\left(\mathbf{r}, \mathbf{r}^{\prime}\right) \cdot \mathbf{J}_{n}\left(\mathbf{r}^{\prime}\right)\right. \\
& \left.\quad+\int_{\partial V_{n}} \mathrm{~d} S^{\prime}\left[\nabla \times \underline{\underline{G}}_{n}\left(\mathbf{r}, \mathbf{r}^{\prime}\right)\right] \cdot \mathbf{M}_{n}\left(\mathbf{r}^{\prime}\right)\right)_{\tan } \\
& \quad=\left(\mathbf{E}_{n}^{\operatorname{inc}}(\mathbf{r})\right)_{\tan }, \quad \mathbf{r} \in \partial V_{n},
\end{aligned}
$$

where the subscript tan denotes the tangential component of the fields. In a similar way, starting from the wave equation for the magnetic field,

$$
\nabla \times \nabla \times \mathbf{H}(\mathbf{r})-k_{n}^{2} \mathbf{H}(\mathbf{r})=\nabla \times \mathbf{j}(\mathbf{r}), \quad \mathbf{r} \in V_{n},
$$

and identifying the incident magnetic field $\mathbf{H}_{n}^{\text {inc }}$ generated by the electrical current density $\mathbf{j}$ inside $V_{n}$,

$$
\mathbf{H}_{n}^{\text {inc }}\left(\mathbf{r}^{\prime}\right)=\int_{V_{n}} \mathrm{~d} V[\nabla \times \mathbf{j}(\mathbf{r})] \cdot \underline{\underline{G}}_{n}\left(\mathbf{r}, \mathbf{r}^{\prime}\right),
$$

one obtains an equation analogous to Eq. (9):

$$
\begin{aligned}
& i \omega \epsilon_{n} \int_{\partial V_{n}} \mathrm{~d} S^{\prime} \underline{\underline{G}}_{n}\left(\mathbf{r}, \mathbf{r}^{\prime}\right) \cdot \mathbf{M}_{n}\left(\mathbf{r}^{\prime}\right) \\
& \quad-\int_{\partial V_{n}} \mathrm{~d} S^{\prime}\left[\nabla \times \underline{\underline{G}}_{n}\left(\mathbf{r}, \mathbf{r}^{\prime}\right)\right] \cdot \mathbf{J}_{n}\left(\mathbf{r}^{\prime}\right) \\
& =\mathbf{H}_{n}^{\text {inc }}(\mathbf{r})- \begin{cases}\mathbf{H}(\mathbf{r}): & \mathbf{r} \in V_{n} \\
0: & \text { otherwise, }\end{cases}
\end{aligned}
$$

leading to the magnetic field integral equation (MFIE)

$$
\begin{aligned}
& \left(i \omega \epsilon_{n} \int_{\partial V_{n}} \mathrm{~d} S^{\prime} \underline{\underline{G}}_{n}\left(\mathbf{r}, \mathbf{r}^{\prime}\right) \cdot \mathbf{M}_{n}\left(\mathbf{r}^{\prime}\right)\right. \\
& \left.\quad-\int_{\partial V_{n}} \mathrm{~d} S^{\prime}\left[\nabla^{\prime} \times \underline{\underline{G}}_{n}\left(\mathbf{r}, \mathbf{r}^{\prime}\right)\right] \cdot \mathbf{J}_{n}\left(\mathbf{r}^{\prime}\right)\right)_{\tan } \\
& \quad=\left(\mathbf{H}_{n}^{\mathrm{inc}}(\mathbf{r})\right)_{\tan }, \quad \mathbf{r} \in \partial V_{n} .
\end{aligned}
$$

The conservation of currents on a boundary $\partial V_{n}=\partial V_{m}$ between two adjacent domains $V_{n}$ and $V_{m}$ requires $\mathbf{J}_{n}$ $=-\mathbf{J}_{m}$ and $\mathbf{M}_{n}=-\mathbf{M}_{m}$ in Eqs. (10) and (14).

Let us now introduce the symmetry of a lattice in $d$ directions $(d=1,2,3)$. A lattice translation vector $\mathbf{t}$ is a linear combination $\mathbf{t}=\Sigma_{i} c_{i} \mathbf{a}_{i}$, with $c_{i} \in \mathbb{Z}$ and $\mathbf{a}_{i}, i=1, \ldots, d$ being the primitive lattice vectors. The unit cell of the lattice is called $\Omega$ (Fig. 1). The irreducible representations of the translation group are defined by the wavevectors $\mathbf{k}$ in the first Brillouin zone [33]. The one-dimensional spaces serving as the basis for the irreducible representations are formed from Bloch functions $\mathbf{U}_{\mathbf{k}}$ satisfying Floquetperiodic boundary conditions:

$$
\mathbf{U}_{\mathbf{k}}(\mathbf{r}-\mathbf{t})=e^{-i \mathbf{k} \cdot \mathbf{t}} \mathbf{U}_{\mathbf{k}}(\mathbf{r}) .
$$

The projections of the incident electric and magnetic fields onto this space are denoted by $\mathbf{E}_{n, \mathbf{k}}^{\text {inc }}$ and $\mathbf{H}_{n, \mathbf{k}}^{\text {inc }}$, respectively. If the source currents $\mathbf{j}$ in Eq. (1) are not Floquet-periodic, one must consider the incident conditions $\mathbf{E}_{n}^{\text {inc }}$ and $\mathbf{H}_{n}^{\text {inc }}$ as a linear combination of Floquetperiodic components $\mathbf{E}_{n, \mathbf{k}}^{\text {inc }}$ and $\mathbf{H}_{n, \mathbf{k}}^{\text {inc }}$.

The computation of the EFIE (10) can be restricted to the boundary surfaces $\partial V_{n}^{\Omega} \equiv \partial V_{n} \cap \Omega$ in the unit cell:

$$
\begin{aligned}
& \left(i \omega \mu_{n} \int_{\partial V_{n}^{\Omega}} \mathrm{d} S^{\prime} \underline{\underline{G}}_{n, \mathbf{k}}\left(\mathbf{r}, \mathbf{r}^{\prime}\right) \cdot \mathbf{J}_{n, \mathbf{k}}\left(\mathbf{r}^{\prime}\right)\right. \\
& \left.\quad+\int_{\partial V_{n}^{\Omega}} \mathrm{d} S^{\prime}\left[\nabla^{\prime} \times \underline{\underline{G}}_{n, \mathbf{k}}\left(\mathbf{r}, \mathbf{r}^{\prime}\right)\right] \cdot \mathbf{M}_{n, \mathbf{k}}\left(\mathbf{r}^{\prime}\right)\right)_{\tan } \\
& =\left(\mathbf{E}_{n, \mathbf{k}}^{\mathrm{inc}}(\mathbf{r})\right)_{\tan }, \quad \mathbf{r} \in \partial V_{n}^{\Omega} .
\end{aligned}
$$

The same projection on the MFIE yields

$$
\begin{aligned}
& \left(i \omega \epsilon_{n} \int_{\partial V_{n}^{\Omega}} \mathrm{d} S^{\prime} \underline{\underline{G}}_{n, \mathbf{k}}\left(\mathbf{r}, \mathbf{r}^{\prime}\right) \cdot \mathbf{M}_{n, \mathbf{k}}\left(\mathbf{r}^{\prime}\right)\right. \\
& \left.\quad-\int_{\partial V_{n}^{\Omega}} \mathrm{d} S^{\prime}\left[\nabla^{\prime} \times \underline{\underline{G}}_{n, \mathbf{k}}\left(\mathbf{r}, \mathbf{r}^{\prime}\right)\right] \cdot \mathbf{J}_{n, \mathbf{k}}\left(\mathbf{r}^{\prime}\right)\right)_{\tan } \\
& \quad=\left(\mathbf{H}_{n, \mathbf{k}}^{\mathrm{inc}}(\mathbf{r})\right)_{\tan }, \quad \mathbf{r} \in \partial V_{n}^{\Omega} .
\end{aligned}
$$

Equations (16) and (17) are solved independently for each Floquet component of the equivalent surface currents $\mathbf{J}_{n, \mathbf{k}}$ and $\mathbf{M}_{n, \mathbf{k}}$. The dyadic $\underline{\underline{G}}_{n, \mathbf{k}}$ is the pseudo-periodic Green's function

$$
\underline{\underline{G}}_{n, \mathbf{k}}\left(\mathbf{r}, \mathbf{r}^{\prime}\right)=\sum_{\mathbf{t}} e^{i \mathbf{k} \cdot \mathbf{t}} \underline{\underline{G}}_{n}\left(\mathbf{r}-\mathbf{t}, \mathbf{r}^{\prime}\right)
$$

It satisfies $\underline{\underline{G}}_{n, \mathbf{k}}\left(\mathbf{r}-\mathbf{t}, \mathbf{r}^{\prime}\right)=e^{-i \mathbf{k} \cdot \mathbf{t}} \underline{\underline{G}}_{n, \mathbf{k}}\left(\mathbf{r}, \mathbf{r}^{\prime}\right)$ and $\underline{\underline{G}}_{n, \mathbf{k}}\left(\mathbf{r}, \mathbf{r}^{\prime}\right)^{\dagger}$ $=\underline{\underline{G}}_{n, \mathbf{k}}\left(\mathbf{r}^{\prime}, \mathbf{r}\right)$, where $\underline{\underline{G}}_{n, \mathbf{k}}\left(\mathbf{r}, \mathbf{r}^{\prime}\right)^{\dagger}$ is the conjugate transpose of $\underline{\underline{G}}_{n, \mathbf{k}}\left(\mathbf{r}, \mathbf{r}^{\prime}\right)$. The equivalent surface currents $\mathbf{J}_{n, \mathbf{k}}$ and $\mathbf{M}_{n, \mathbf{k}}$, solutions of the EFIE and MFIE, are not the actual currents flowing on the surfaces $\partial V_{n}^{\Omega}$, but they produce the same electromagnetic field inside the regions $V_{n}^{\Omega}$. From the first case in Eqs. (9) and (13), expressions for the electric and magnetic fields scattered by the objects at any point $\mathbf{r} \in V_{n}$ can be derived as 


$$
\begin{aligned}
\mathbf{E}_{n, \mathbf{k}}^{\mathrm{scat}}(\mathbf{r})= & -i \omega \mu_{n} \int_{\partial V_{n}^{\Omega}} \mathrm{d} S^{\prime} \underline{\underline{G}}_{n, \mathbf{k}}\left(\mathbf{r}, \mathbf{r}^{\prime}\right) \cdot \mathbf{J}_{n, \mathbf{k}}\left(\mathbf{r}^{\prime}\right) \\
& -\int_{\partial V_{n}^{\Omega}} \mathrm{d} S^{\prime}\left[\nabla^{\prime} \times \underline{\underline{G}}_{n, \mathbf{k}}\left(\mathbf{r}, \mathbf{r}^{\prime}\right)\right] \cdot \mathbf{M}_{n, \mathbf{k}}\left(\mathbf{r}^{\prime}\right), \\
\mathbf{H}_{n, \mathbf{k}}^{\mathrm{scat}}(\mathbf{r})= & -i \omega \epsilon_{n} \int_{\partial V_{n}^{\Omega}} \mathrm{d} S^{\prime} \underline{\underline{G}}_{n, \mathbf{k}}\left(\mathbf{r}, \mathbf{r}^{\prime}\right) \cdot \mathbf{M}_{n, \mathbf{k}}\left(\mathbf{r}^{\prime}\right) \\
& +\int_{\partial V_{n}^{\Omega}} \mathrm{d} S^{\prime}\left[\nabla^{\prime} \times \underline{\underline{G}}_{n, \mathbf{k}}\left(\mathbf{r}, \mathbf{r}^{\prime}\right)\right] \cdot \mathbf{J}_{n, \mathbf{k}}\left(\mathbf{r}^{\prime}\right) .
\end{aligned}
$$

For simplicity, the labels $\mathbf{k}$ and $\Omega$ are omitted in the following.

\section{NUMERICAL IMPLEMENTATION}

Details on the numerical implementation of the EFIE (16) and MFIE (17) are given in this section. They require the evaluation of the periodic Green's function, a timeconsuming step without the use of an accelerating technique such as Ewald's method. Hence, the application of Ewald's method to this problem is given. It is followed by the discretization of the EFIE and MFIE with the MoM for an arbitrary number of regions and boundary surfaces. For periodic systems, this requires the implementation of specific boundary conditions if the scatterer completely fills the unit cell.

\section{A. Evaluation of the Periodic Green's Function with Ewald's Method}

Although the integral equations (16) and (17) can be applied for periodicities in one, two, or three directions, we shall now focus on the most common physical situation and assume that the regions $V_{n}$ carry the symmetry of a two-dimensional lattice with primitive translation vectors $\mathbf{a}_{1}$ and $\mathbf{a}_{2}$. Details about the implementation of Ewald's method for periodicities in one and three directions can be found in, e.g., [34-36], respectively. The area of the unit cell is $|\Omega|=\left|\mathbf{a}_{1} \times \mathbf{a}_{2}\right|$. Let us define $\mathbf{R}=\mathbf{r}-\mathbf{r}^{\prime}$ and $\mathbf{R}_{\mathbf{t}}=\mathbf{R}-\mathbf{t}$ with $\mathbf{t}=c_{1} \mathbf{a}_{1}+c_{2} \mathbf{a}_{2}$ and $c_{1}, c_{2} \in \mathbb{Z}$. The periodic dyadic Green's function (18) depends on $\mathbf{R}$ only:

$$
\underline{\underline{G}}_{n}\left(\mathbf{r}, \mathbf{r}^{\prime}\right)=\left(\underline{\underline{1}}+\frac{\nabla \nabla}{k_{n}^{2}}\right) G_{n}\left(\mathbf{r}, \mathbf{r}^{\prime}\right)
$$

where

$$
G_{n}\left(\mathbf{r}, \mathbf{r}^{\prime}\right)=\sum_{\mathbf{t}} \frac{e^{i k_{n}\left|\mathbf{R}_{\mathbf{t}}\right|}}{4 \pi\left|\mathbf{R}_{\mathbf{t}}\right|} e^{i \mathbf{k} \cdot \mathbf{t}}
$$

In fact, Eqs. (16) and (17) can be transformed following [25] to involve the evaluation of the Green's function (22) and its gradient only. The infinite sum of Eq. (22) converges slowly and requires many terms to reach a reasonable accuracy. Using Ewald's method [30,37], the Green's function can be transformed into two rapidly converging sums:

$$
G_{n}=G_{n}^{(1)}+G_{n}^{(2)}
$$

where $G_{n}^{(2)}$ is a sum over lattice vectors $\mathbf{t}$ :

$$
G_{n}^{(2)}\left(\mathbf{r}, \mathbf{r}^{\prime}\right)=\frac{1}{8 \pi} \sum_{\mathbf{t}} e^{i \mathbf{k} \cdot \mathbf{t}} \sum_{ \pm} \frac{e^{ \pm i k_{n}\left|\mathbf{R}_{\mathbf{t}}\right|}}{\left|\mathbf{R}_{\mathbf{t}}\right|} \operatorname{erfc}\left(\left|\mathbf{R}_{\mathbf{t}}\right| E \pm \frac{i k_{n}}{2 E}\right)
$$

The sum $G_{n}^{(1)}$ is in reciprocal space. The reciprocal lattice vectors $\mathbf{b}_{1}$ and $\mathbf{b}_{2}$ are given by

$$
\mathbf{b}_{1}=2 \pi \frac{\mathbf{a}_{2} \times\left(\mathbf{a}_{1} \times \mathbf{a}_{2}\right)}{\left|\mathbf{a}_{1} \times \mathbf{a}_{2}\right|^{2}}, \quad \mathbf{b}_{2}=2 \pi \frac{\mathbf{a}_{1} \times\left(\mathbf{a}_{2} \times \mathbf{a}_{1}\right)}{\left|\mathbf{a}_{1} \times \mathbf{a}_{2}\right|^{2}} .
$$

Defining the vectors $\mathbf{u}=\Sigma_{i} d_{i} \mathbf{b}_{i}$, with $d_{i} \in \mathbb{Z}$, one obtains for $G_{n}^{(1)}$

$G_{n}^{(1)}\left(\mathbf{r}, \mathbf{r}^{\prime}\right)=\frac{1}{4|\Omega|} \sum_{\mathbf{u}} e^{i(\mathbf{k}-\mathbf{u}) \cdot \mathbf{R}} \sum_{ \pm} \frac{e^{ \pm \gamma_{n, \mathbf{k}, \mathbf{u}} R_{\perp}}}{\gamma_{n, \mathbf{k}, \mathbf{u}}} \operatorname{erfc}\left(\frac{\gamma_{n, \mathbf{k}, \mathbf{u}}}{2 E} \pm R_{\perp} E\right)$,

with $\gamma_{n, \mathbf{k}, \mathbf{u}}=\sqrt{|\mathbf{k}-\mathbf{u}|^{2}-k_{n}^{2}}$. The variable $R_{\perp}$ is the component of $\mathbf{R}$ in the direction normal to both $\mathbf{a}_{1}$ and $\mathbf{a}_{2}$. The value of the splitting parameter $E$ is discussed in the following. Due to the complementary error functions, the sums in Eq. (23) ensure a Gaussian convergence rate for the evaluation of the periodic Green's function and its gradient.

The best choice $E_{0}$ for the splitting parameter $E$ is the one that balances the decay rate of the two series $G_{n}^{(1)}$ and $G_{n}^{(2)}$ [30]. Good results are obtained for $E=\sqrt{\pi /|\Omega|}$. For large periodic spacing (or equivalently high wavenumber $k_{n}$ ) the complementary error functions in Eqs. (26) and (24) take large imaginary arguments, and their summation suffers from high accuracy losses. This problem can be avoided by requiring that $k_{n}^{2} /\left(4 E^{2}\right)$ should be smaller than a maximum permitted exponent $H^{2}$ [30]. In such a case, a higher number of terms are required to reach an acceptable relative error in the evaluation. The optimal parameter is finally chosen to be $E_{0}$ $=\max \left(\sqrt{\pi /|\Omega|}, k_{n} / 2 H\right)$.

\section{B. Solution by Method of Moments}

A technique for solving the EFIE (16) and the MFIE (17) is the MoM [26]. The equivalent surface currents are expanded in terms of Rao-Wilton-Glisson (RWG) basis functions $\mathbf{f}_{i}^{n}$, building a triangular mesh approximating the boundary surface $\partial V_{n}$ [38]:

$$
\begin{array}{r}
\mathbf{J}_{n}=\sum_{i} \alpha_{i} \mathbf{f}_{i}^{n}, \\
\mathbf{M}_{n}=\sum_{i} \beta_{i} \mathbf{f}_{i}^{n},
\end{array}
$$

where the index $i$ labels the different edges within all regions (Fig. 1). If two RWG functions $\mathbf{f}_{i}^{n}$ and $\mathbf{f}_{i}^{n^{\prime}}$ are associated with the same edge, the conservation of current on $\partial V_{n}=\partial V_{n^{\prime}}$ between the two adjacent regions $V_{n}$ and $V_{n^{\prime}}$ implies $\mathbf{f}_{i}^{n}=-\mathbf{f}_{i}^{n^{\prime}}$ (Fig. 2(a); see also [39]). If more than two regions are touching an edge, all the expansion coefficients related to this edge are identified [Fig. 2(b)]. In this 

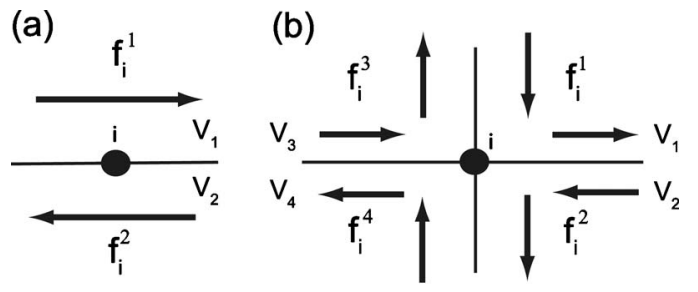

(c)

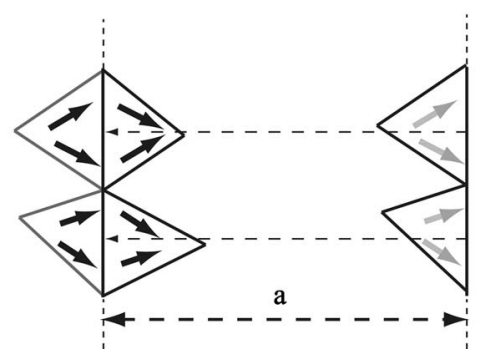

Fig. 2. (a) Conservation of current across the boundary between regions $V_{1}$ and $V_{2}$ implies for the RWG functions $\mathbf{f}_{i}^{1}$ and $\mathbf{f}_{i}^{2}$ associated with the same edge $i$ to have opposite signs. (b) This property can be generalized to an arbitrary number of boundaries associated with the same edge. (c) If the discretization reaches opposite ends of the unit cell, currents on these borders are not linearly independent. The mesh has to be translation symmetric and one border is removed from the calculations.

case, more than one RWG function is associated with the same edge, but only one per adjacent region. If the region $V_{n}$ is not adjacent to the edge $i$, then $\mathbf{f}_{i}^{n} \equiv 0$. Similarly to the FEM, the Galerkin method is applied, multiplying Eqs. (16) and (17) with the basis functions and integrating over $\partial V_{n}$. Defining the sets $\{\alpha\}$ and $\{\beta\}$ of expansion coefficients $\alpha_{i}$ and $\beta_{i}$, the EFIE (16) can be rewritten as a matrix equation for $\{\alpha\}$ and $\{\beta\}$ :

$$
\left[\begin{array}{ll}
i \omega \mu_{n} \mathbf{D}^{n} & \mathbf{K}^{n}
\end{array}\right] \cdot\left[\begin{array}{l}
\{\alpha\} \\
\{\beta\}
\end{array}\right]=\mathbf{q}^{(E), n},
$$

with submatrices

$$
\begin{gathered}
D_{i j}^{n}=\int_{\partial V_{n}} \mathrm{~d} S \mathbf{f}_{i}^{n}(\mathbf{r}) \cdot \int_{\partial V_{n}} \mathrm{~d} S^{\prime} \underline{\underline{G}}_{n}\left(\mathbf{r}, \mathbf{r}^{\prime}\right) \cdot \mathbf{f}_{j}^{n}\left(\mathbf{r}^{\prime}\right), \\
K_{i j}^{n}=\int_{\partial V_{n}} \mathrm{~d} S \mathbf{f}_{i}^{n}(\mathbf{r}) \cdot \int_{\partial V_{n}} \mathrm{~d} S^{\prime}\left[\nabla^{\prime} \times \underline{\underline{G}}_{n}\left(\mathbf{r}, \mathbf{r}^{\prime}\right)\right] \cdot \mathbf{f}_{j}^{n}\left(\mathbf{r}^{\prime}\right), \\
q_{i}^{(E), n}=\int_{\partial V_{n}} \mathrm{~d} S \mathbf{f}_{i}^{n}(\mathbf{r}) \cdot \mathbf{E}_{n}^{\text {inc }}(\mathbf{r}) .
\end{gathered}
$$

A similar matrix equation can be found for the MFIE (17):

$$
\left[\begin{array}{ll}
\mathbf{K}^{n} & -i \omega \epsilon_{n} \mathbf{D}^{n}
\end{array}\right] \cdot\left[\begin{array}{l}
\{\alpha\} \\
\{\beta\}
\end{array}\right]=\mathbf{q}^{(H), n},
$$

with

$$
q_{i}^{(H), n}=-\int_{\partial V_{n}} \mathrm{~d} S \mathbf{f}_{i}^{n}(\mathbf{r}) \cdot \mathbf{H}_{n}^{\mathrm{inc}}(\mathbf{r}) .
$$

Integrals (30) and (31) can be computed numerically using Gaussian quadrature [40]. The periodic Green's func- tion and its gradient are evaluated using the results of Subsection 3.A. In some cases, solving for $\{\alpha\}$ and $\{\beta\}$ with the EFIE or the MFIE does not result in the same values. In fact, especially in resonant conditions, the results may also exhibit large errors due to poor testing. The PoggioMiller-Chang-Harrigton-Wu-Tsai (PMCHWT) formulation combines the EFIE and MFIE to solve them simultaneously [41]. Although the PMCHWT formulation might lead to poor conditioning of the system matrix and a slow convergence of iterative solvers [22], it has proven to give stable and accurate results [42,43], even in resonant conditions [25]. In that case, Eqs. (29) and (33) are combined for all regions, summing over $n$ :

$$
\left[\begin{array}{cc}
\sum_{n} i \omega \mu_{n} \mathbf{D}^{n} & \sum_{n} \mathbf{K}^{n} \\
\sum_{n} \mathbf{K}^{n} & -\sum_{n} i \omega \epsilon_{n} \mathbf{D}^{n}
\end{array}\right] \cdot\left[\begin{array}{l}
\{\alpha\} \\
\{\beta\}
\end{array}\right]=\sum_{n}\left[\begin{array}{l}
\mathbf{q}^{(E), n} \\
\mathbf{q}^{(H), n}
\end{array}\right] .
$$

Equation (35) can be solved for $\{\alpha\}$ and $\{\beta\}$ to obtain the values of the equivalent surface currents $\mathbf{J}$ and $\mathbf{M}$ flowing on the interfaces between different media. The scattered electric and magnetic fields can then be obtained in each region $V_{n}$ from $\{\alpha\}$ and $\{\beta\}$. Inserting decompositions (27) and (28) in Eqs. (19) and (20),

$$
\begin{aligned}
\mathbf{E}_{n}^{\text {scat }}(\mathbf{r})= & \sum_{i}-i \omega \mu_{n} \int_{\partial V_{n}} \mathrm{~d} S^{\prime} \underline{\underline{G}}_{n}\left(\mathbf{r}, \mathbf{r}^{\prime}\right) \cdot \alpha_{i} \mathbf{f}_{i}^{n}\left(\mathbf{r}^{\prime}\right) \\
& -\int_{\partial V_{n}} \mathrm{~d} S^{\prime}\left[\nabla^{\prime} \times \underline{\underline{G}}_{n}\left(\mathbf{r}, \mathbf{r}^{\prime}\right)\right] \cdot \beta_{i} \mathbf{f}_{i}^{n}\left(\mathbf{r}^{\prime}\right), \\
\mathbf{H}_{n}^{\text {scat }}(\mathbf{r})= & \sum_{i}-i \omega \epsilon_{n} \int_{\partial V_{n}} \mathrm{~d} S^{\prime} \underline{\underline{G}}_{n}\left(\mathbf{r}, \mathbf{r}^{\prime}\right) \cdot \beta_{i} \mathbf{f}_{i}^{n}\left(\mathbf{r}^{\prime}\right) \\
& +\int_{\partial V_{n}} \mathrm{~d} S^{\prime}\left[\nabla^{\prime} \times \underline{\underline{G}}_{n}\left(\mathbf{r}, \mathbf{r}^{\prime}\right)\right] \cdot \alpha_{i} \mathbf{f}_{i}^{n}\left(\mathbf{r}^{\prime}\right) .
\end{aligned}
$$

The matrix elements of Eqs. (30) and (31) can be turned into integrals involving the scalar Green's function $G_{n}\left(\mathbf{r}, \mathbf{r}^{\prime}\right)$ or its gradient in their integrand, which is known to be divergent for $\left|\mathbf{r}^{\prime}-\mathbf{r}\right| \rightarrow 0$. This behavior of the Green's function can also lead to inaccurate results in the numerical evaluation of the matrix elements relative to neighboring triangles. An elegant way to overcome this difficulty is to separate the Green's function into a singular part that can be integrated in a closed form and a smooth slowly varying part that can be accurately integrated numerically (see the Appendix for more details). Highly conductive metals with Green's function approaching a Dirac distribution can therefore be handled accurately. The same procedure can be repeated for Eqs. (36) and (37), which guarantees an accurate field evaluation close to the scatterer surface.

If the discretized object completely fills the unit cell, the discretization mesh must be translation symmetric on opposite edges, in order to allow a continuity of the current flowing across the unit cell [Fig. 2(c)]. The periodic boundary condition (15) imposes a constraint on the equivalent surface currents: 


$$
\begin{aligned}
\mathbf{J}_{n}(\mathbf{r}-\mathbf{t}) & =e^{-i \mathbf{k} \cdot \mathbf{t}} \mathbf{J}_{n}(\mathbf{r}), \\
\mathbf{M}_{n}(\mathbf{r}-\mathbf{t}) & =e^{-i \mathbf{k} \cdot \mathbf{t}} \mathbf{M}_{n}(\mathbf{r}) .
\end{aligned}
$$

Currents on opposite borders are not linearly independent. If two edges are separated from each other by a lattice vector, their associated expansion coefficients have to be identified. The RWG function associated with the resulting edge is defined over the existing border triangle inside the unit cell and a translation of the triangle attached to the opposite discarded edge [Fig. 2(c)].

\section{APPLICATIONS OF THE SIE METHOD TO PERIODIC NANOSTRUCTURES}

The SIE method features a high flexibility in the simulation of homogeneous scatterers with frequency-dependent dielectric permittivity or magnetic permeability. The integral equations presented in Sections 2 and 3 are formulated for an arbitrary number of regions or scatterers, and can therefore model the scattering on periodic composite objects with any shape. In fact, this approach also broadens the variety of structures that can be simulated thanks to an appropriate use of the periodic symmetries. In particular, when the periodicity is along two directions, one or several of the scatterers' surfaces can fill the unit cell such as in Figs. 3 and 4 to create periodic structures in stratified media. This represents another attractive feature of a SIE approach. The two-dimensional periodic symmetry can be merged to create two-dimensional structures periodic in one direction, such as gratings.

Since it represents the most common physical situation, the periodicity in two directions is illustrated with three examples in this section. In Subsection 4.A, the case of a plane wave incident on an infinite planar interface is compared to the analytical solution both in near- and farfields, for metallic and high permittivity scatterers and for several types of skewed lattices. In Subsection 4.B, the photonic bandgap properties of a square array of pillars are investigated using far-field reflectance calculations

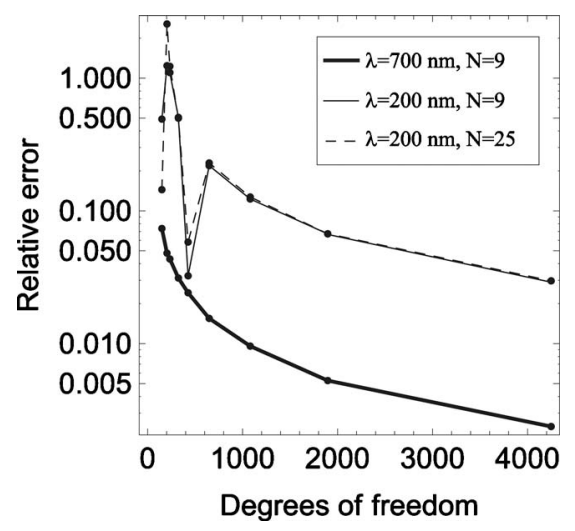

Fig. 3. Relative error on the transmittance through a planar infinite interface between air and a material with a relative dielectric permittivity of 2 . The incident field in air is an $s$-polarized plane wave with a $45^{\circ}$ incidence angle. Results are compared to the analytical solution and a relative error is computed as a function of the DOFs for different wavelengths $\lambda=200 \mathrm{~nm}$ and $\lambda$ $=700 \mathrm{~nm}$, and different numbers of terms $N$ in Ewald's sum. The unit cell has dimensions $500 \mathrm{~nm} \times 500 \mathrm{~nm}$. and near-field sampling. The effect of a substrate is also revealed by these calculations. In Subsection 4.C, the effective refractive index of a metamaterial is computed and is shown to be negative in the red part of the optical spectrum.

\section{A. Planar Infinite Interface}

As a first example, the scatterer surface is a single square completely filling the unit cell, mimicking an infinite planar interface between two media with different permittivities. To assess the accuracy of the method, the transmittance for an $s$-polarized plane wave at $45^{\circ}$ incidence is computed and compared in Fig. 3 to the analytical solution for different numbers of degrees of freedom (DOFs). The number of DOFs is twice the number of edges in the mesh since two unknowns are associated with each edge. The linear system of equations is solved with conjugate gradients [44]. The SIE formulation presented for individual scatterers in [25] has been proven to be more accurate than VIE methods. Comparing Fig. 3 and Fig. 3 of [25], the number of DOFs per unit area required to reach a given level of accuracy is comparable (for $0.02 \%$ error, approximately $5.8 \times 10^{-3} \mathrm{DOF} / \mathrm{nm}^{2}$ in [25] for a dielectric sphere, and approximately $2.4 \times 10^{-3} \mathrm{DOF} / \mathrm{nm}^{2}$ in our case for an incidence wavelength of $700 \mathrm{~nm}$ ). This shows that the SIE/PMCHWT formulation presented here has the same accuracy as that presented in [25]. The simulations are also performed for different numbers of terms in the evaluation of the Green's function with Ewald's method. In this case, this parameter plays a negligible role in the accuracy of the results, even for a relatively high frequency incident field.

In Fig. 4, the physical situation is the same but several simulation parameters are changed. The instantaneous scattered electric field is computed from Eqs. (36) and (37) and compared to the analytical solution for a plane wave in vacuum at normal incidence. In Fig. 4(a), the plane wave impinges on a metallic surface with relative permittivity $\epsilon=-17+i$. In Fig. 4(b), the surface is made from a high permittivity $\epsilon=14$ material. In Figs. 4(a) and 4(b) the interface is modeled by a squared flat scatterer. The field evaluation is performed in the near-field and a high accuracy is observed, even at distances shorter than $1 \mathrm{~nm}$ from the interface. In Fig. 4(c), the discretized object is a parallelogram and the corresponding lattice is skewed. Very good agreement is also obtained in this situation. A calculation of the electric field in the far-field zone is shown, for example, $5 \mu \mathrm{m}$ from the surface in Fig. 4(c). In all cases, this excellent agreement is obtained in reflection and transmission for a reasonable number of DOFs (with an average triangle edge size of $10 \mathrm{~nm}$ ).

\section{B. Square Array of Pillars}

The reflection properties of a square array of pillars of refractive index $n=3.36$ as a function of the incident wavelength $\lambda$ are investigated in Fig. 5. The periodic Green's function is evaluated with nine terms in the Ewald summation. The linear system of equations is also solved with conjugate gradients [44]. From Eq. (19) the intensity of the scattered electric field $\left|\mathbf{E}^{\text {scat }}\right|^{2}$ is calculated $4 \mu \mathrm{m}$ above the array on the incidence side and normalized to the intensity of the incident plane wave $\left|\mathbf{E}^{\text {inc }}\right|^{2}$, yielding 
(a)

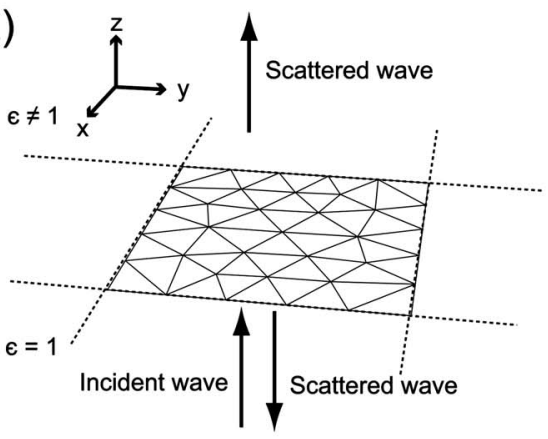

(c)

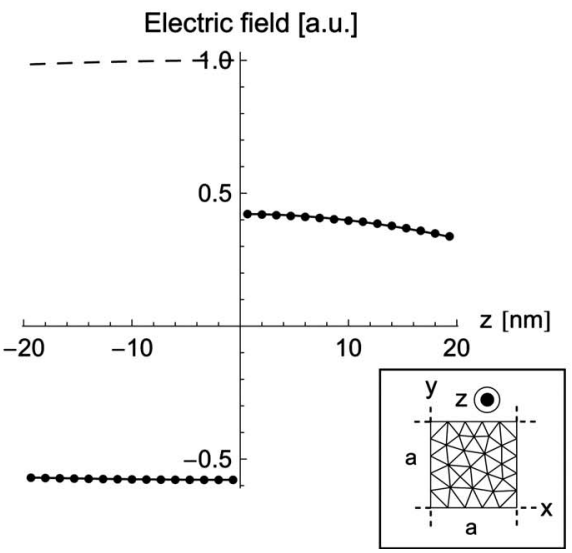

(b)

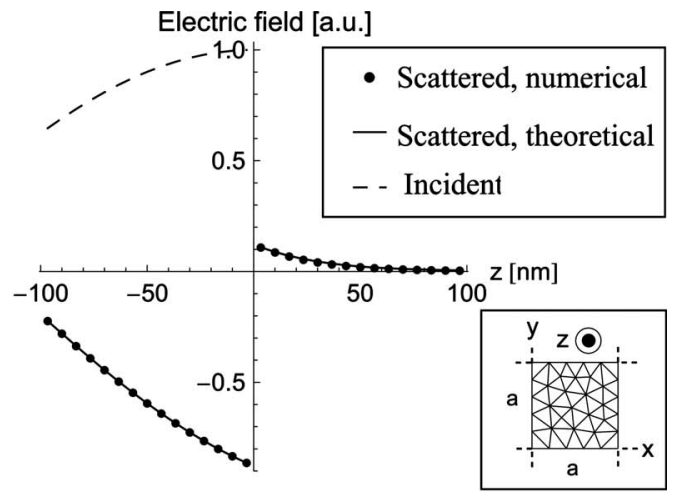

(d)

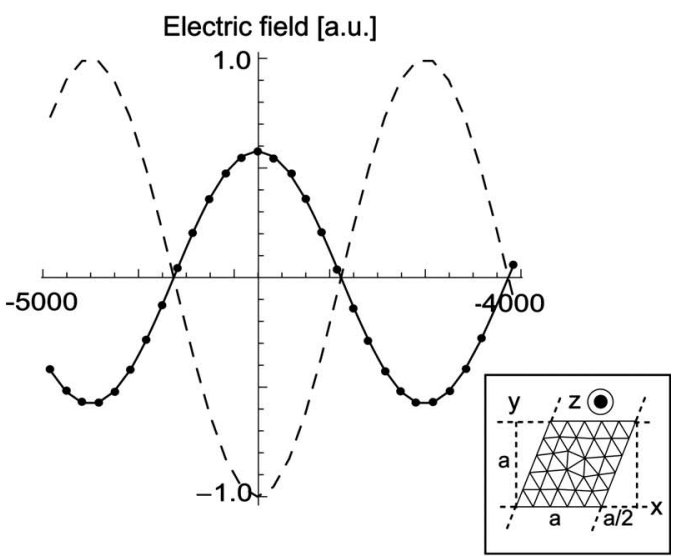

Fig. 4. Plane-wave scattering at normal incidence on a planar infinite interface between two dielectric media. (a) Discretization of the interface and incidence conditions. (b) Air-metal interface $\left[\epsilon=(-17+i) \epsilon_{0}\right]$. (c), (d) Air-high-permittivity dielectric interface $\left(\epsilon=14 \epsilon_{0}\right)$. The tangential component of the instantaneous electric field is calculated along the incidence direction and compared to the analytical solution. The lattice period is $a=50 \mathrm{~nm}$. Insets of (b)-(d): discretized objects used for the calculations.

(a)
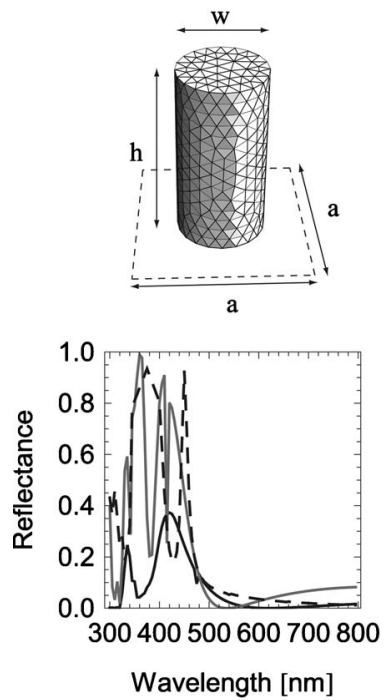

(b)
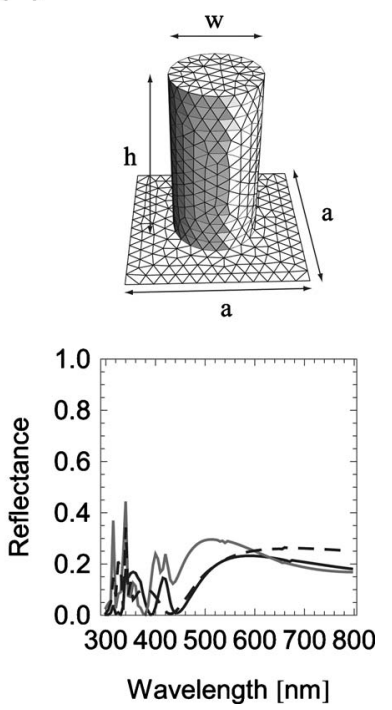

Fig. 5. Reflectance of a square array of pillars with a refractive index of 3.36 and dimensions $w=100 \mathrm{~nm}$ and $h=200 \mathrm{~nm}$. The lattice period is $a=200 \mathrm{~nm}$. Two cases are compared for normal (solid black curve), $45^{\circ} p$-polarized (solid gray curve), and $45^{\circ}$ $s$-polarized (dashed curve) plane-wave incidences: (a) without substrate (758 mesh triangles) and (b) with a substrate of the same material (968 mesh triangles).

the reflectance of the system. The pillars without a substrate display a very high reflectance (up to unity) between $\lambda=340 \mathrm{~nm}$ and $\lambda=460 \mathrm{~nm}$ for both $p$-polarized and $s$-polarized incidences, indicating the position of a full photonic bandgap [Fig. 5(a)]. This effect is detailed in Fig. 6 (a) showing a standing wave in the reflection region and almost no amplitude in the transmission region. The strong gradient of electric field polarization in the array also indicates that the wave cannot propagate in the structure. However, a normal incidence wave is less reflected due to the finite thickness of the array. For larger wavelengths the system is almost transparent [Figs. 5(a) and $6(\mathrm{~b})]$. In this case, the wave is unchanged between the reflection and the transmission regions, and lights up between the pillars to satisfy Maxwell's equations' boundary conditions.

If the array lies on a substrate, the interface between air and the substrate must also be discretized [Fig. 5(b)]. When a plane wave impinges the system from the air region, the reflectance reaches only 0.55 for $p$-polarization and 0.76 for $s$-polarization. The high reflectance band is centered at $\lambda=340 \mathrm{~nm}$ with a width of $40 \mathrm{~nm}$. Comparing these results with Fig. 5(a), we see that the photonic bandgap is drastically reduced both in amplitude and bandwidth. Waves in the array now leak to the substrate due to the finite thickness of the pillars and can therefore penetrate even within the bandgap [Fig. 6(c)]. The transmitted wave reproduces the array geometry, while a 


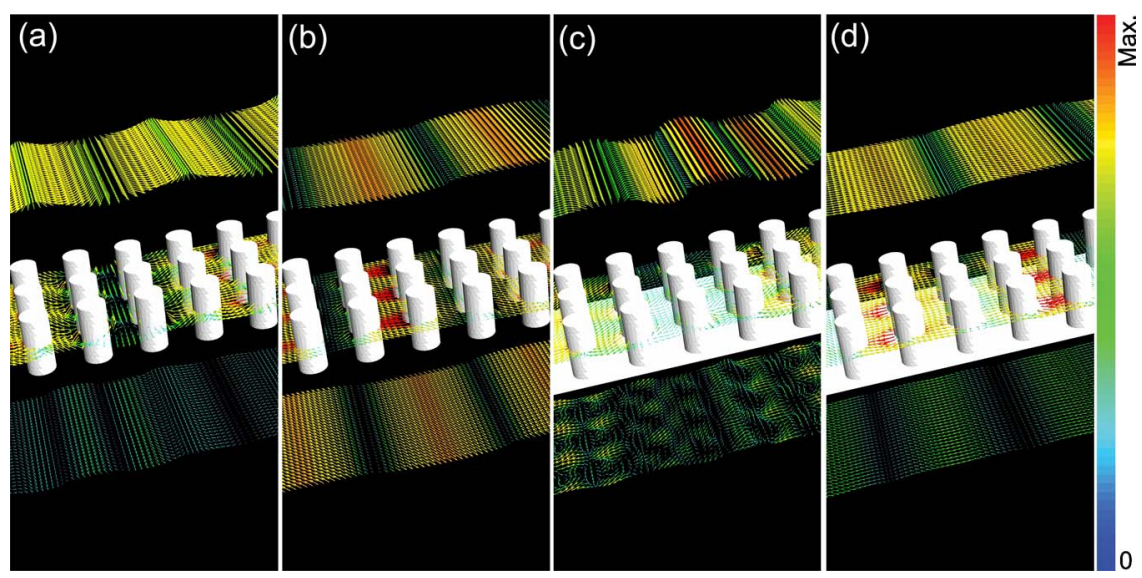

Fig. 6. (Color online) Photonic crystal made with an infinite square array of pillars with a refractive index of 3.36 . The real part of the total (incident+scattered) instantaneous electric field is calculated in planes at $500 \mathrm{~nm}$ above, $500 \mathrm{~nm}$ below, and in the array for a $45^{\circ}$ $p$-polarized plane wave incident from above. The scale is normalized in each frame. The arrow length is proportional to the electric field. (a),(b) No substrate [cf. Fig. 5(a)]; (c),(d) with substrate [cf. Fig. 5(b)]. Different illumination wavelengths $\lambda$ are considered: (a) $\lambda=350 \mathrm{~nm}$, (b) $\lambda=700 \mathrm{~nm},(\mathrm{c}) \lambda=340 \mathrm{~nm}$, (d) $\lambda=700 \mathrm{~nm}$.

standing wave is observed in the air region. A strong gradient of the electric field polarization is observed in the array where wave propagation is forbidden. For longer wavelengths, the array is almost transparent and scattering resembles Fresnel refraction [Figs. 5(b) and 6(d)].

In order to check the validity of the results in Fig. 5, we have performed two additional analytical tests that are commonly used in grating theories [1]. Results are given in Table 1. The first of them is the energy balance. From Eqs. (19) and (20) the time-averaged Poynting vector $\mathbf{P}^{\text {scat }}=1 / 2 \mathbf{E}^{\text {scat }} \times\left(\mathbf{H}^{\text {scat }}\right)^{*}$ is evaluated. For any surface $S$ enclosing a non-lossy material volume it must satisfy $E$ $=\oint \mathrm{d} S \hat{\mathbf{n}} \cdot \mathbf{P}^{\text {scat }}(\mathbf{r})=\mathbf{0}$, where $\hat{\mathbf{n}}$ is the surfaces' outward pointing normal vector. The surface $S$ has been chosen here to be a rectangular box of section $\Omega$ and height $10 \mu \mathrm{m}$ centered on a pillar. From Eq. (15), the energy balance is automatically satisfied on the sides of $S$. Therefore, the flux $E$ can be evaluated only $5 \mu \mathrm{m}$ away from the scatterers on surfaces above and below the array. The second test involves reciprocity. Let $\mathbf{E}_{1}$ and $\mathbf{H}_{1}$ denote the scattered electric and magnetic fields of a given diffraction problem. Consider another diffraction problem by choosing a propagating reflected order of the first problem and returning an incident field of the same wavelength along the direction of this outgoing order. The scattered electric and magnetic fields of the second problem are denoted by $\mathbf{E}_{2}$ and $\mathbf{H}_{2}$. Considering again a surface $S$ enclos-

Table 1. Energy Balance $E$ and Reciprocity $R$ for the Square Array of Pillars (See Text) ${ }^{a}$

\begin{tabular}{lccc}
\hline Geometry & Incident Polarization & $E /\left(2|\Omega| Z_{0}\right)$ & $R /\left(2|\Omega| Z_{0}\right)$ \\
\hline Figure 5(a) & Normal & $4 \times 10^{-3}$ & $1 \times 10^{-2}$ \\
& $s$ & $1 \times 10^{-3}$ & $5 \times 10^{-3}$ \\
& $p$ & $2 \times 10^{-3}$ & $3 \times 10^{-3}$ \\
Figure 5(b) & Normal & $1 \times 10^{-1}$ & - \\
& $s$ & $5 \times 10^{-3}$ & $1 \times 10^{-1}$ \\
& $p$ & $7 \times 10^{-3}$ & $6 \times 10^{-2}$ \\
\hline
\end{tabular}

${ }^{a}$ The quantities $|\Omega|$ and $Z_{0}$ denote the unit cell's area and the free space impedance, respectively. ing the array, reciprocity implies $R=\oint \mathrm{d} S \hat{\mathbf{n}} \cdot\left(\mathbf{E}_{\mathbf{1}} \times \mathbf{H}_{\mathbf{2}}-\mathbf{E}_{\mathbf{2}}\right.$ $\left.\times \mathbf{H}_{1}\right)(\mathbf{r})=\mathbf{0}$. The total fluxes $E$ and $R$ are numerically nonzero and have been normalized to $2|\Omega| Z_{0}$, where $Z_{0}$ is the free space impedance. For the geometry in Fig. 5(a) [respectively, Fig. 5(b)], we have chosen the zeroth order transmitted [respectively, reflected] wave as the second diffraction problem. In all cases, the numerical error exponentially decreases as a function of the incident wavelength. Table 1 shows the errors obtained at $\lambda=500 \mathrm{~nm}$, corresponding to a mesh size of a tenth of the effective wavelength. Very good agreement is obtained (less than $1 \%$ error), but the accuracy decreases when the pillars lie on the substrate of high dielectric permittivity.

\section{Negative-Index Metamaterial}

We compare in this subsection the results of our method to the published results of Dolling et al. [45] where they experimentally and numerically demonstrated a negative-index metamaterial operating around a $\lambda$ $=780 \mathrm{~nm}$ wavelength. The geometry shown in Fig. 7(a) and materials are directly taken from [45]. The metamaterial is made from a $\mathrm{Ag}-\mathrm{MgF}_{2}-\mathrm{Ag}$ multilayer. The system of equations is solved with the LU decomposition package LAPACK [46]. The refractive index of $\mathrm{MgF}_{2}$ is 1.38, and the Drude model with a plasma frequency of $1.37 \times 10^{16} \mathrm{~s}^{-1}$ and damping of $9 \times 10^{13} \mathrm{~s}^{-1}$ is taken for silver. The incident plane wave is $p$-polarized with the propagation vector in the $x-z$ plane, making an angle of $6^{\circ}$ with the normal to the array. In order to retrieve the effective refractive index, a homogeneous film with a thickness and complex transmission and reflection coefficients identical to those of the metamaterial is considered [47]. Very good agreement with the results of [45] is obtained in Fig. 7(b), showing a negative effective refractive index of the metamaterial for wavelengths between 786 and 796 $\mathrm{nm}$. The index reaches -0.3 at $790 \mathrm{~nm}$, for a figure of merit of 0.4 .

\section{SUMMARY}

A surface integral formulation for light scattering on periodic structures has been presented. We have derived the 
(a)

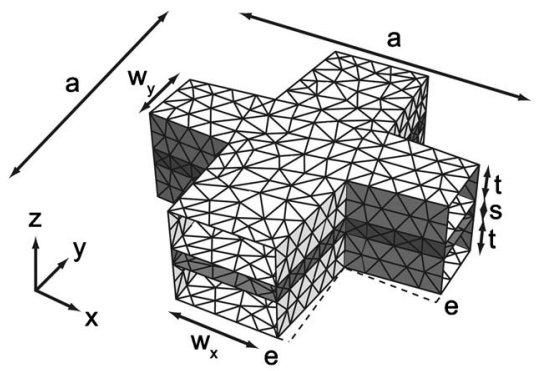

(b)

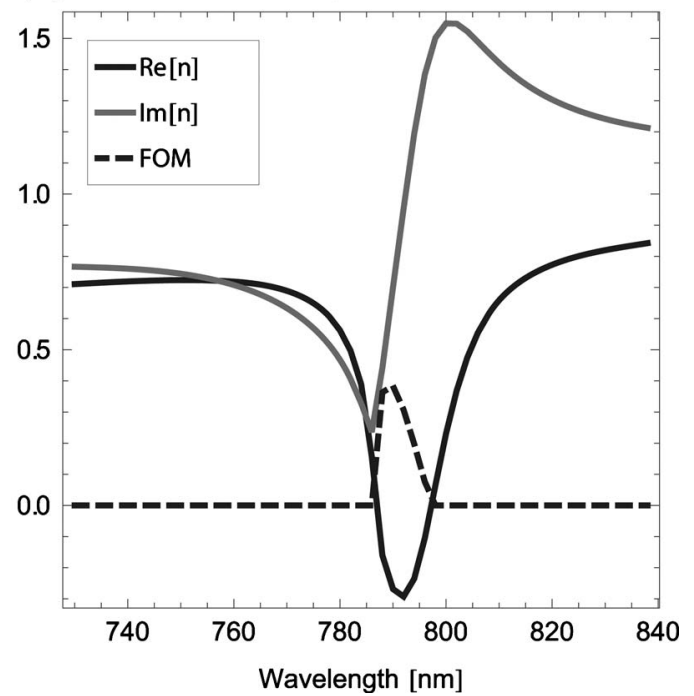

Fig. 7. (a) Geometry of the metamaterial's unit cell (1352 mesh triangles): period $a=300 \mathrm{~nm}$, Ag layer thickness $t=40 \mathrm{~nm}, \mathrm{MgF}_{2}$ layer thickness $s=17 \mathrm{~nm}$, deviation from rectangular shape $e$ $=8 \mathrm{~nm}$, width $w_{x}=102 \mathrm{~nm}$, and $w_{y}=102 \mathrm{~nm}$. (b) Metamaterial's effective refractive index $n$ as a function of the wavelength: real and imaginary parts and figure of merit $\mathrm{FOM}=-\operatorname{Re}(n) / \operatorname{Im}(n)$. The FOM is set to zero if $\operatorname{Re}(n)$ is positive.

electric and magnetic field equations on the scatterers' surfaces in the unit cell with periodic boundary conditions and detailed their numerical implementation into two main steps: the evaluation of the periodic Green's function with Ewald's method and the solution computation with the method of moments (MoM). This technique has then been applied to the scattering on a infinite planar interface between air and metals or high permittivity materials. Calculated results have been compared to the analytical solution and have shown very good agreement. In a second example, we have investigated the near- and farfield optical properties of a photonic crystal made of a square array of dielectric pillars. We have shown that these properties can be strongly affected by the presence of a substrate. As a final illustration of the technique, we have calculated the refractive index of a fishnet metamaterial and retrieved a negative refractive index in the red region of the optical spectrum, in good agreement with published experimental data.

With this versatile formulation, a very large variety of geometries can be simulated, including doubly periodic structures on substrates and in multilayered media. The surface discretization provides a high flexibility, allowing the investigation of irregular shapes, including fabrication accuracy. Another advantage of the formulation is that it can give insight into the extreme near-field of the scatterers, while providing at the same time the corre- sponding far-field. This method shall find numerous applications for the design of realistic photonic and plasmonic [48] nanostructures, in which light propagation is tailored to produce novel optical effects.

\section{APPENDIX: REGULARIZATION SCHEME}

The integrand in matrix elements (30) and (31) relative to the same triangle element diverges. Inaccurate results can also be obtained for matrix elements relative to neighboring triangles. Following [49] [Eqs. (6) and (20)], the matrix elements (30) can be transformed to

$$
\begin{aligned}
D_{i j}^{n}= & \int_{\partial V_{n}} \mathrm{~d} S \mathbf{f}_{i}^{n}(\mathbf{r}) \cdot\left(\underline{\underline{1}}+\frac{\nabla \nabla}{k_{n}^{2}}\right) \cdot \int_{\partial V_{n}} \mathrm{~d} S^{\prime} G_{n}\left(\mathbf{r}, \mathbf{r}^{\prime}\right) \cdot \mathbf{f}_{j}^{n}\left(\mathbf{r}^{\prime}\right) \\
= & \frac{1}{k_{n}^{2}} \int_{\partial V_{n}} \mathrm{~d} S \mathbf{f}_{i}^{n}(\mathbf{r}) \cdot \nabla \int_{\partial V_{n}} \mathrm{~d} S^{\prime} G_{n}\left(\mathbf{r}, \mathbf{r}^{\prime}\right) \nabla^{\prime} \cdot \mathbf{f}_{j}^{n}\left(\mathbf{r}^{\prime}\right) \\
& +\int_{\partial V_{n}} \mathrm{~d} S \mathbf{f}_{i}^{n}(\mathbf{r}) \cdot \int_{\partial V_{n}} \mathrm{~d} S^{\prime} G_{n}\left(\mathbf{r}, \mathbf{r}^{\prime}\right) \mathbf{f}_{j}^{n}\left(\mathbf{r}^{\prime}\right) \\
= & -\frac{1}{k_{n}^{2}} \int_{\partial V_{n}} \mathrm{~d} S \nabla \cdot \mathbf{f}_{i}^{n}(\mathbf{r}) \int_{\partial V_{n}} \mathrm{~d} S^{\prime} G_{n}\left(\mathbf{r}, \mathbf{r}^{\prime}\right) \nabla^{\prime} \cdot \mathbf{f}_{j}^{n}\left(\mathbf{r}^{\prime}\right) \\
& +\int_{\partial V_{n}} \mathrm{~d} S \mathbf{f}_{i}^{n}(\mathbf{r}) \cdot \int_{\partial V_{n}} \mathrm{~d} S^{\prime} G_{n}\left(\mathbf{r}, \mathbf{r}^{\prime}\right) \mathbf{f}_{j}^{n}\left(\mathbf{r}^{\prime}\right)
\end{aligned}
$$

Using the identity $\nabla^{\prime} \times \underline{\underline{G}}_{n}\left(\mathbf{r}, \mathbf{r}^{\prime}\right)=-\left[\nabla \underline{\underline{G}}_{n}\left(\mathbf{r}, \mathbf{r}^{\prime}\right)\right] \times \underline{\underline{1}}$, the matrix elements (31) become

$$
\begin{aligned}
K_{i j}^{n} & =\int_{\partial V_{n}} \mathrm{~d} S \mathbf{f}_{i}^{n}(\mathbf{r}) \cdot \int_{\partial V_{n}} \mathrm{~d} S^{\prime}\left[\nabla^{\prime} \times \underline{\underline{G}}_{n, \mathbf{k}}\left(\mathbf{r}, \mathbf{r}^{\prime}\right)\right] \cdot \mathbf{f}_{j}^{n}\left(\mathbf{r}^{\prime}\right) \\
& =\int_{\partial V_{n}} \mathrm{~d} S \mathbf{f}_{i}^{n}(\mathbf{r}) \cdot \int_{\partial V_{n}} \mathrm{~d} S^{\prime}\left[\nabla^{\prime} G_{n}\left(\mathbf{r}, \mathbf{r}^{\prime}\right)\right] \times \mathbf{f}_{j}^{n}\left(\mathbf{r}^{\prime}\right)
\end{aligned}
$$

The lattice sum $G_{n}^{(2)}$ [Eq. (24)] has a singularity for $|\mathbf{R}|$ $\rightarrow 0$. The Green's function and its gradient can be separated into a singular part that can be integrated in a closed form [49] and a smooth slowly varying part that can be accurately integrated numerically:

$$
G_{n}\left(\mathbf{r}, \mathbf{r}^{\prime}\right)=G_{n}^{(s)}(\mathbf{R})+\frac{1}{4 \pi}\left(\frac{1}{|\mathbf{R}|}-\frac{k_{n}^{2}|\mathbf{R}|}{2}\right)
$$

where $G_{n}^{(s)}\left(\mathbf{r}, \mathbf{r}^{\prime}\right)$ is non-singular and differentiable for $|\mathbf{R}| \rightarrow 0$. The following formulas around the singularity can be used:

$$
\begin{aligned}
\lim _{R \rightarrow 0} & {\left[\sum_{ \pm} \frac{e^{ \pm i k R}}{R} \operatorname{erfc}\left(R E \pm \frac{i k}{2 E}\right)-\frac{2}{R}\right] } \\
& =-\frac{4 E}{\sqrt{\pi}} e^{k^{2} / 4 E^{2}}+2 i k\left[\operatorname{erfc}\left(\frac{i k}{2 E}-1\right)\right],
\end{aligned}
$$




$$
\begin{aligned}
\lim _{R \rightarrow 0}[ & -\frac{4 E}{R \sqrt{\pi}} e^{-R^{2} E^{2}+k^{2} / 4 E^{2}} \\
& \left.+\sum_{ \pm} \frac{-1 \pm i k R}{R^{2}} e^{ \pm i k R} \operatorname{erfc}\left(R E \pm \frac{i k}{2 E}\right)+\frac{2}{R^{2}}\right]=-2 k^{2} .
\end{aligned}
$$

\section{ACKNOWLEDGMENTS}

It is a pleasure to acknowledge stimulating discussions with M. Schnieper and A. Stuck. This work was supported by the Competence Center for Materials Science and Technology (CCMX) under grant Zonop and by the Swiss National Science Foundation (SNSF) under grant 20021116758.

\section{REFERENCES}

1. R. Petit, Electromagnetic Theory of Gratings (SpringerVerlag, 1980).

2. J. D. Joannopoulos, P. R. Villeneuve, and S. H. Fan, "Photonic crystals: Putting a new twist on light," Nature 386, 143-149 (1997).

3. C. M. Soukoulis, S. Linden, and M. Wegener, "Negative refractive index at optical wavelengths," Science 315, 47-49 (2007).

4. S. Bozhevolnyi, J. Erland, K. Leosson, P. Skovgaard, and J. Hvam, "Waveguiding in surface plasmon polariton band gap structures," Phys. Rev. Lett. 86, 3008-3011 (2001).

5. L. Li, "New formulation of the Fourier modal method for crossed surface-relief gratings," J. Opt. Soc. Am. A 14, 2758-2767 (1997).

6. K. Sakoda, Optical Properties of Photonic Crystals (Springer, 2005).

7. M. G. Moharam, E. B. Grann, D. A. Pommet, and T. K. Gaylord, "Formulation for stable and efficient implementation of the rigorous coupled-wave analysis of binary gratings," J. Opt. Soc. Am. A 12, 1068-1076 (1995).

8. D. Whittaker and I. Culshaw, "Scattering-matrix treatment of patterned multilayer photonic structures," Phys. Rev. B 60, 2610-2618 (1999).

9. S. Tikhodeev, A. Yablonskii, E. Muljarov, N. Gippius, and T. Ishihara, "Quasiguided modes and optical properties of photonic crystal slabs," Phys. Rev. B 66, 045102 (2002).

10. K. Yee, "Numerical solution of initial boundary value problems involving Maxwell's equations in isotropic media," IEEE Trans. Antennas Propag. 14, 302-307 (1966).

11. P. Monk, Finite Element Methods for Maxwell's Equations (Oxford University Press, 2003).

12. J. Jin, Finite Element Method in Electromagnetics (Wiley, 2002).

13. J. Chandezon, D. Maystre, and G. Raoult, "A new theoretical method for diffraction gratings and its numerical application,” J. Opt. Nouv. Rev. Opt. 11, 235-241 (1980).

14. A. Wirgin and R. Deleuil, "Theoretical and experimental investigation of a new type of blazed grating," J. Opt. Soc. Am. 59, 1348-1357 (1969).

15. T. Delort and D. Maystre, "Finite-element method for gratings," J. Opt. Soc. Am. A 10, 2592-2601 (1993).

16. B. T. Draine and P. J. Flatau, "Discrete-dipole approximation for scattering calculations," J. Opt. Soc. Am. A 11, 1491-1499 (1994).

17. O. J. F. Martin and N. B. Piller, "Electromagnetic scattering in polarizable backgrounds," Phys. Rev. E 58, 3909-3915 (1998).

18. P. C. Chaumet, A. Rahmani, and G. W. Bryant, "Generalization of the coupled dipole method to periodic structures," Phys. Rev. B 67, 165404 (2003).

19. B. T. Draine and P. J. Flatau, "Discrete-dipole approxima- tion for periodic targets: theory and tests," J. Opt. Soc. Am. A 25, 2693-2703 (2008).

20. P. C. Chaumet and A. Sentenac, "Simulation of light scattering by multilayer cross-gratings with the coupled dipole method," J. Quant. Spectrosc. Radiat. Transf. 110, 409-414 (2009).

21. T. Eibert, J. Volakis, D. Wilton, and D. Jackson, "Hybrid FE/BI modeling of 3-D doubly periodic structures utilizing triangular prismatic elements and an MPIE formulation accelerated by the Ewald transformation," IEEE Trans. Antennas Propag. 47, 843-850 (1999).

22. M. S. Yeung and E. Barouch, "Three-dimensional nonplanar lithography simulation using a periodic fast multipole method," Proc. SPIE 3051, 509-521 (1997).

23. M. S. Yeung, "Single integral equation for electromagnetic scattering by three-dimensional homogeneous dielectric objects," IEEE Trans. Antennas Propag. 47, 1615-1622 (1999).

24. F. J. García de Abajo and A. Howie, "Retarded field calculation of electron energy loss in inhomogeneous dielectrics," Phys. Rev. B 65, 115418 (2002).

25. A. M. Kern and O. J. F. Martin, "Surface integral formulation for $3 \mathrm{D}$ simulations of plasmonic and high permittivity nanostructures," J. Opt. Soc. Am. A 26, 732-740 (2009).

26. R. F. Harrington, Field Computation by Moment Methods (Macmillan, 1968).

27. N. Marly, D. De Zutter, and H. Pues, "A surface integral equation approach to the scattering and absorption of doubly periodic lossy structures," IEEE Trans. Electromagn. Compat. 36, 14-22 (1994).

28. N. Marly, B. Baekelandt, D. De Zutter, and H. Pues, "Integral equation modeling of the scattering and absorption of multilayered doubly-periodic lossy structures," IEEE Trans. Antennas Propag. 43, 1281-1287 (1995).

29. L. Trintinalia and H. Ling, "Integral equation modeling of multilayered doubly-periodic lossy structures using periodic boundary condition and a connection scheme," IEEE Trans. Antennas Propag. 52, 2253-2261 (2004).

30. I. Stevanovic, P. Crespo-Valero, K. Blagovic, F. Bongard, and J. R. Mosig, "Integral-equation analysis of 3-D metallic objects arranged in 2-D lattices using the Ewald transformation," IEEE Trans. Microwave Theory Tech. 54, 36883697 (2006).

31. H. Fischer, A. Nesci, G. Leveque, and O. J. F. Martin, "Characterization of the polarization sensitivity anisotropy of a near-field probe using phase measurements," J. Microsc. 230, 27-31 (2008).

32. C.-T. Tai, Dyadic Green Functions in Electromagnetic Theory, IEEE Series on Electromagnetic Waves, 2nd ed. (IEEE, 1994).

33. W. Ludwig and C. Falter, Symmetries in Physics: Group Theory Applied to Physical Problems, Vol. 64 of Springer Series in Solid-State Sciences (Springer-Verlag, 1988).

34. A. Rathsfeld, G. Schmidt, and B. H. Kleemann, "On a fast integral equation method for diffraction gratings," Comm. Comp. Phys. 1, 984-1009 (2006).

35. F. Capolino, D. R. Wilton, and W. A. Johnson, "Efficient computation of the 3D Green's function for the Helmholtz operator for a linear array of point sources using the Ewald method," J. Comput. Phys. 223, 250-261 (2007).

36. I. Stevanoviæ and J. R. Mosig, "Periodic Green's function for skewed 3-D lattices using the Ewald transformation," Microwave Opt. Technol. Lett. 49, 1353-1357 (2007).

37. K. E. Jordan, G. R. Richter, and P. Sheng, "An efficient numerical evaluation of the green-function for the Helmholtz operator on periodic structures," J. Comput. Phys. 63, 222235 (1986).

38. S. Rao, D. Wilton, and A. Glisson, "Electromagnetic scattering by surfaces of arbitrary shape," IEEE Trans. Antennas Propag. 30, 409-418 (1982).

39. P. Ylä-Oijala, M. Taskinen, and J. Sarvas, "Surface integral equation method for general composite metallic and dielectric structures with junctions," PIER 52, 81-108 (2005).

40. G. R. Cowper, "Gaussian quadrature formulas for triangles," Int. J. Numer. Methods Eng. 7, 405-408 (1973).

41. T. K. Wu and L. L. Tsai, "Scattering from arbitrarily-shaped 
lossy dielectric bodies of revolution," Radio Sci. 12, 709-718 (1977).

42. X. Q. Sheng, J. M. Jin, J. M. Song, W. C. Chew, and C. C. $\mathrm{Lu}$, "Solution of combined-field integral equation using multilevel fast multipole algorithm for scattering by homogeneous bodies," IEEE Trans. Antennas Propag. 46, 17181726 (1998).

43. P. Ylä-Oijala and M. Taskinen, "Application of combined field integral equation for electromagnetic scattering by dielectric and composite objects," IEEE Trans. Antennas Propag. 53, 1168-1173 (2005).

44. P. J. Flatau, "Improvements in the discrete-dipole approximation method of computing scattering and absorption," Opt. Lett. 22, 1205-1207 (1997).

45. G. Dolling, M. Wegener, C. M. Soukoulis, and S. Linden, "Negative-index metamaterial at $780 \mathrm{~nm}$ wavelength," Opt. Lett. 32, 53-55 (2007).
46. E. Anderson, Z. Bai, C. Bischof, S. Blackford, J. Demmel, J. Dongarra, J. Du Croz, A. Greenbaum, S. Hammarling, A. McKenney, and D. Sorensen, LAPACK Users' Guide, 3rd ed. (Society for Industrial and Applied Mathematics, 1999).

47. D. R. Smith, S. Schultz, P. Markos, and C. M. Soukoulis, "Determination of effective permittivity and permeability of metamaterials from reflection and transmission coefficients," Phys. Rev. B 65, 195104 (2002).

48. B. Gallinet and O. J. F. Martin, "Scattering on plasmonic nanostructures arrays modeled with a surface integral formation," Photonics Nanostruct. Fund. Appl. 8, 278-284 (2010).

49. I. Hanninen, M. Taskinen, and J. Sarvas, "Singularity subtraction integral formulae for surface integral equations with RWG, rooftop and hybrid basis functions," PIER $\mathbf{6 3}$, 243-278 (2006). 